\title{
Relativistic Alpha Field Theory - Part II: Does a Gravitational Field Could be Without Singularity?
}

\author{
Branko M. Novakovic
}

\begin{abstract}
General Relativity Theory (GRT) cannot be applied to the extremely strong gravitational field at the Planck's scale, because of the related singularity. Here we show that Relativistic Alpha Field (RAF) theory extends the application of GRT to the extremely strong fields at the Planck's scale. This is the consequence of the following predictions of RAF theory: a) no a singularity at the Schwarzschild radius, b) there exists a minimal radius at $r=r_{\min }=\left(G M / 2 c^{2}\right)$ that prevents singularity at $r=0$, i.e. the nature protects itself, c) the gravitational force becomes positive (repulsive) if $\left(G M / r c^{2}\right)>1$, that could be a source of a dark energy, and d) unification of electrical and gravitational forces can be done in the standard four dimensions (4D). Predictions a) and b) are presented in this (second) part of this theory. It has been shown that the metrics of the line element is regular in the region where radius is greater or equal to $\boldsymbol{r}_{\min }$ and less than infinity. The predictions c) and d) are considered in the third part of the theory. The key point for the predictions of RAF theory is the solution of the field parameters presented in the first part of the theory. If RAF theory is correct, then it could be applied to the both weak and strong fields at the Universe and Planck's scales giving the new light to the regions like black holes, quantum theory, high energy physics, Big Bang theory and cosmology.
\end{abstract}

Kay words: Relativistic alpha field theory, Energy momentum tensors, Electrical field, Gravitational field

\section{INTRODUCTION}

As it is well known, General Relativity Theory (GRT) [1-6] cannot be applied to the extremely strong gravitational field at the Planck's scale, because of the related singularity. Here we present a new theory that is called Relativistic Alpha Field (RAF) theory. We show that RAF theory extends the capability of the GRT for the application to the extremely strong fields at the Planck's scale. This is the consequence of the following predictions of RAF theory: a) no a singularity at the Schwarzschild radius, b) there exists a minimal radius at $r$ $=\left(G M / 2 c^{2}\right)$ that prevents singularity at $r=0$, i.e. the nature protects itself, c) the gravitational force becomes positive (repulsive) if $\left(G M / r c^{2}\right)>1$, that could be a source of a dark energy in the universe, and d) unification of electrical and gravitational forces can be done in the standard four dimensions (4D). Predictions a) and b) are presented in this (second) part of this theory. It has been shown that the metrics of the line element is regular in the region where radius is greater or equal to $r_{\text {min }}$ and less than infinity. This means that the metrics of the line element is regular at the Schwarzschild radius as well as at the minimal radius. This proves predictions a) and b) of RAF theory. The predictions c) and d) are considered in the third part of the theory.

Branko Novakovic, FSB - University of Zagreb, Luciceva 5, P.O.B. 509, 10000 Zagreb, Croatia,
The key point for predictions of RAF theory is the solution of the field parameters presented in the first part of the theory. This solution provides derivation of the energy-momentum tensor for the electrical and gravitational fields as well as their unified field, using of the geometric approach. Further, we show that the mentioned field parameters satisfy the Einstein's field equations with a cosmological constant $\Lambda=$ 0 . In the case of a strong static gravitational field, the quadratic term $\left(G M / r c^{2}\right)^{2}$ generates the related energy-momentum tensor $T_{\mu \eta}$ for the static field. For that case we do not need to add by hand the related energy-momentum tensor $T_{\mu \eta}$ on the right side of the Einstein's field equations. In the case of a weak static gravitational field, like in our solar system, the quadratic term $\left(G M / r c^{2}\right)^{2}$ is close to zero and can be neglected. For that case the field parameters satisfy the Einstein's field equations in a vacuum $\left(T_{\mu \eta}=0, \Lambda=0\right)$.

It is also well known, that for unification of the electroweak and strong interactions with gravity, one can use the following two possibilities [1-6]: a) trying to describe gravity as a gauge theory, or b) trying to describe gauge theories as gravity. The first possibility (a) has attracted a lot of attention, but because of the known difficulties, this approach set gravity apart from the standard gauge theories. The second possibility (b) is much more radical. The initial idea has been proposed by Kaluza-Klein theory [7, 8], which today has many variations [9-14], and takes the place in the modern theories like high energy physics (supergravity [15-17] and string theories [18-29]). These theories use five or more extra dimensions with the related dimensional reduction to the four dimensions. Meanwhile, we do not know the answers to the some questions like: can we take the extra dimensions as a real, or as a mathematical device? Following the solution of the two dimensionless (unitless) field parameters $\alpha$ and $\alpha^{\prime}$ for unified electrical and gravitational field in the first part of RAF theory [30], the unification of electrical and gravitational forces in the standard four dimensions (4D) has been presented in the third part of RAF theory [31]. This unification is based on the geometric approach.

RAF theory starts with the main preposition: if the electrical, gravitational and unified fields (forces) can be described by the geometric approach, then the field parameters $\alpha$ and $\alpha^{\prime}$ of a particle in the electrical, gravitational and unified fields should satisfy the Einstein's field equations and the Einstein's geodesic equations. The proposition, related to the satisfaction of the Einstein's field equations is proved in this (second) part of RAF theory. The proposition, related to the satisfaction of the Einstein's geodesic equations is proved in the third part [31] of RAF theory. If RAF theory is correct, then it could be applied to the both weak and strong fields at the Universe and Planck's scales giving the new light to the regions like black holes, quantum theory, high energy physics, Big Bang theory and cosmology. 
This paper is organized as follows. Derivation of the energy-momentum tensor for electrical and gravitational fields is presented in Sec. II. The proofs of the predictions a) and b) of RAF theory is also presented in Sec. II, as a subsection A. Sec. III shows the procedure of derivation of energy-momentum tensor for unified electrical and gravitational field. Finally, the related conclusion and reference list are presented in Sec. IV and Sec. V, respectively.

\section{ENERGY-MOMENTUM TENSOR FOR ELECTRICAL AND GRAVITATIONAL FIELDS}

The basic problem of this paper is to determine the energy-momentum tensors for electrical, gravitational and unified field in the Einstein's four dimension (4D), by using the gravity (geometric) concept. In that sense we started with the general line element $d s^{2}$ in an alpha field, given in the first part [30] of this theory

$$
\begin{gathered}
d s^{2}=-\alpha \alpha^{\prime} c^{2} d t^{2}-\kappa\left(\alpha-\alpha^{\prime}\right)_{x} c d t d x-\kappa\left(\alpha-\alpha^{\prime}\right)_{y} c d t d y \\
-\kappa\left(\alpha-\alpha^{\prime}\right)_{z} c d t d z+d x^{2}+d y^{2}+d z^{2} .
\end{gathered}
$$

Following the well known procedure [1-6], this line element can be transformed into the spherical polar coordinates in the nondiagonal form

$$
\begin{aligned}
d s^{2}=- & \alpha \alpha^{\prime} c^{2} d t^{2}-\kappa\left(\alpha-\alpha^{\prime}\right) c d t d r+d r^{2} \\
& +r^{2} d \theta^{2}+r^{2} \sin ^{2} \theta d \phi^{2} .
\end{aligned}
$$

The line element (2) belongs to the well known form of the Riemann's type line element [32, 33-35]

$$
\begin{gathered}
d s^{2}=g_{00}\left(d x^{0}\right)^{2}+2 g_{01} d x^{0} d x^{1}+g_{11}\left(d x^{1}\right)^{2} \\
+g_{22}\left(d x^{2}\right)^{2}+g_{33}\left(d x^{3}\right)^{2} .
\end{gathered}
$$

Comparing the equations (2) and (3) we obtain the coordinates and components of the covariant metric tensor, valid for the line element (2):

$$
\begin{aligned}
& d x^{0}=c d t, \quad d x^{1}=d r, \quad d x^{2}=d \theta, \quad d x^{3}=d \phi, \\
& g_{00}=-\alpha \alpha^{\prime}, \quad g_{01}=g_{10}=\frac{-\kappa\left(\alpha-\alpha^{\prime}\right)}{2}, \\
& g_{11}=1, \quad g_{22}=r^{2}, \quad g_{33}=r^{2} \sin ^{2} \theta .
\end{aligned}
$$

Starting with the line element (2) we employ, for the convenient, the following substitutions:

$$
v=\alpha \alpha^{\prime}, \quad \lambda=\kappa\left(\alpha^{\prime}-\alpha\right) / 2 \text {. }
$$

In that case the nondiagonal line element (2) is transformed into the new relation

$$
\begin{aligned}
d s^{2}= & -v c^{2} d t^{2}+2 \lambda c d t d r+d r^{2} \\
& +r^{2} d \theta^{2}+r^{2} \sin ^{2} \theta d \phi^{2} .
\end{aligned}
$$

Using the coordinate system (4), the related covariant metric tensor $g_{\mu \eta}$ of the line element (6) is presented by the matrix form

$$
\left[g_{\mu \eta}\right]=\left[\begin{array}{rccc}
-\nu & \lambda & 0 & 0 \\
\lambda & 1 & 0 & 0 \\
0 & 0 & r^{2} & 0 \\
0 & 0 & 0 & r^{2} \sin ^{2} \theta
\end{array}\right] .
$$

This tensor is symmetric and has six non-zero elements as we expected that should be. The contravariant metric tensor $g^{\mu \eta}$ of the nondiagonal line element (6), is derived by inversion of the covariant one (7)

$$
\left[g^{\mu \eta}\right]=\left[\begin{array}{cccc}
-1 /\left(v+\lambda^{2}\right) & \lambda /\left(v+\lambda^{2}\right) & 0 & 0 \\
\lambda /\left(v+\lambda^{2}\right) & v /\left(v+\lambda^{2}\right) & 0 & 0 \\
0 & 0 & 1 / r^{2} & 0 \\
0 & 0 & 0 & 1 / r^{2} \sin ^{2} \theta
\end{array}\right] .
$$

The determinants of the tensors (7) and (8) are given by the relations:

$$
\begin{aligned}
& \operatorname{det}\left[g_{\mu \eta}\right]=-r^{4}\left(v+\lambda^{2}\right) \sin ^{2} \theta, \\
& \operatorname{det}\left[g^{\mu \eta}\right]=-\left(\frac{1}{r^{4}\left(\nu+\lambda^{2}\right) \sin ^{2} \theta}\right) .
\end{aligned}
$$

(a) Proposition 1. If the electrostatic field is described by the line element (6), then the solution of the Einstein field equations gives the energy momentum tensor, $T_{\mu \eta}$, of that field in the following form:

$$
\begin{aligned}
T_{\mu \eta}= & \left(T_{00}, T_{01}, T_{10}, T_{11}, T_{22}, T_{33}\right) \\
= & \left(v,-\lambda,-\lambda,-1, r^{2}, r^{2} \sin ^{2} \theta\right) \frac{\left(G_{e} Q\right)^{2}}{8 \pi G r^{4}}, \\
& G_{e}=\frac{q}{m_{0}}, \quad A_{0}=\frac{Q}{r} .
\end{aligned}
$$

Here $q$ and $m_{0}$ are an electric charge and a rest mass of the electron, while $A_{0}$ is a scalar potential and $Q$ is an electric point charge of the electrostatic field. Parameter $G_{e}=q / m_{0}$ is a constant that remands us to the constant of motion in the geodesic equation of the Kaluza-Klein theory [7-14].

(b) Proof of the proposition 1. In order to prove of the proposition 1, we can start with the second type of the Christoffel symbols of the metric tensors (7) and (8). These symbols can be calculated by employing the well known relation $[1-6]$

$\Gamma_{\eta \mu}^{\gamma}=\frac{g^{\kappa \gamma}}{2}\left[g_{\kappa \eta, \mu}+g_{\kappa \mu, \eta}-g_{\eta \mu, \kappa}\right], \kappa, \gamma, \eta, \mu=0,1,2,3$.

Thus, employing (6), (7), (8) and (11), we obtain the second type Christoffel symbols of the spherically symmetric non-rotating body: 


$$
\begin{aligned}
& \Gamma_{00}^{0}=\left(\dot{v}+2 \lambda \dot{\lambda}+v^{\prime} \lambda\right) / D, \Gamma_{01}^{0}=\Gamma_{10}^{0}=v^{\prime} / D, \Gamma_{11}^{0}=-2 \lambda^{\prime} / D, \\
& \Gamma_{22}^{0}=-2 \lambda r / D, \Gamma_{33}^{0}=\left(-2 \lambda r \sin ^{2} \theta\right) / D, \Gamma_{00}^{1}=\left(-\dot{v} \lambda+2 v \dot{\lambda}+v v^{\prime}\right) / D, \\
& \Gamma_{01}^{1}=\Gamma_{10}^{1}=-v^{\prime} \lambda / D, \Gamma_{11}^{1}=2 \lambda^{\prime} \lambda / D, \Gamma_{22}^{1}=-2 v r / D, \\
& \Gamma_{33}^{1}=\left(-2 v r \sin ^{2} \theta\right) / D, \Gamma_{12}^{2}=\Gamma_{21}^{2}=\frac{1}{r}, \Gamma_{33}^{2}=-\sin \theta \cos \theta, \Gamma_{13}^{3}=\Gamma_{31}^{3}=\frac{1}{r}, \\
& \Gamma_{23}^{3}=\Gamma_{32}^{3}=\operatorname{ctg} \theta, \quad D=2\left(v+\lambda^{2}\right), \frac{\partial v}{\partial t}=\dot{v}, \frac{\partial v}{\partial r}=v^{\prime}, \frac{\partial \lambda}{\partial t}=\dot{\lambda}, \frac{\partial \lambda}{\partial r}=\lambda^{\prime} .
\end{aligned}
$$

For a static field the Christoffel symbols $\Gamma_{00}^{0}$ and $\Gamma_{00}^{1}$ are reduced to the simplest form:

$$
\Gamma_{00}^{0}=\frac{v^{\prime} \lambda}{2\left(v+\lambda^{2}\right)}, \Gamma_{00}^{1}=\frac{v v^{\prime}}{2\left(v+\lambda^{2}\right)}, \frac{\partial v}{\partial r}=v^{\prime}, \frac{\partial \lambda}{\partial r}=\lambda^{\prime} .
$$

In a static field, the other Christoffel symbols in (12) are remaining unchanged.

As it is well known, the determinant of the metric tensor of the line element (6) should satisfy the following condition [33-35]

$$
\sqrt{-\operatorname{det}\left[g_{\mu \eta}\right]}=\sqrt{r^{4}\left(v+\lambda^{2}\right) \sin ^{2} \theta}=1 \text {. }
$$

Including the normalization of the radius, $r=1$, and the angle $\theta=90^{\circ}$ in (14) we obtain the important relations between the parameters $v$ and $\lambda$ :

$$
\begin{aligned}
& v+\lambda^{2}=1, v=1-\lambda^{2}, \\
& v^{\prime}=-2 \lambda \lambda^{\prime}, v^{\prime \prime}=-2\left(\lambda^{\prime 2}+\lambda \lambda^{\prime \prime}\right) .
\end{aligned}
$$

If we take into account the relations (15), then the Christoffel symbols in (12) and (13) become the functions only of the parameter $\lambda$.

For calculation of the related components of the Riemannian tensor $R_{\beta \eta \mu}^{\kappa}$ and Ricci tensor $R_{\eta \mu}$ of the line element (6) we can employ the following relations [1-6]:

$$
\begin{aligned}
& R_{\beta \eta \mu}^{\kappa}=\Gamma_{\beta \mu, \eta}^{\kappa}-\Gamma_{\beta \eta, \mu}^{\kappa}+\Gamma_{\sigma \eta}^{\kappa} \Gamma_{\beta \mu}^{\sigma}-\Gamma_{\sigma \mu}^{\kappa} \Gamma_{\beta \eta}^{\sigma}, \\
& R_{\eta \mu}=R_{\eta \kappa \mu}^{\kappa}=R_{\mu \eta}, \kappa, \beta, \eta, \mu, \sigma=0,1,2,3 .
\end{aligned}
$$

Applying the Christoffel symbols (12) to the relations (16) we obtain the related Ricci tensor for the static field of the line element (6), with the following components:

$$
\begin{aligned}
& R_{00}=-\left(1-\lambda^{2}\right)\left(\lambda^{\prime 2}+\lambda \lambda^{\prime \prime}+\frac{2 \lambda \lambda^{\prime}}{r}\right), \\
& R_{01}=R_{10}=-\lambda\left(\lambda^{\prime 2}+\lambda \lambda^{\prime \prime}+\frac{2 \lambda \lambda^{\prime}}{r}\right), \\
& R_{11}=\left(\lambda^{\prime 2}+\lambda \lambda^{\prime \prime}+\frac{2 \lambda \lambda^{\prime}}{r}\right), R_{22}=2 \lambda \lambda^{\prime} r+\lambda^{2}, \\
& R_{33}=\left(2 \lambda \lambda^{\prime} r+\lambda^{2}\right) \sin ^{2} \theta .
\end{aligned}
$$

The other components of the Ricci tensor are equal to zero. The related Ricci scalar for the static field is determined by the equation

$$
\begin{aligned}
& R=g^{\mu \eta} R_{\mu \eta}, \quad \mu, \eta=0,1,2,3, \rightarrow \\
& R=2\left(\lambda^{\prime 2}+\lambda \lambda^{\prime \prime}+\frac{2 \lambda \lambda^{\prime}}{r}\right)+2\left(\frac{2 \lambda \lambda^{\prime}}{r}+\frac{\lambda^{2}}{r^{2}}\right)
\end{aligned}
$$

In order to calculate the energy-momentum tensor $T_{\mu \eta}$ for the static field, one should employ Ricci tensor (17), Ricci scalar (18) and the Einstein's field equations [1-6] without a cosmological constant $(\Lambda=0)$

$$
R_{\mu \eta}-\frac{1}{2} g_{\mu \eta} R=k T_{\mu \eta}, \quad k=\frac{8 \pi G}{c^{4}}, \mu, \eta=0,1,2,3 .
$$

Here $G$ is the Newton's gravitational constant, $c$ is the speed of the light in a vacuum and $T_{\mu \eta}$ is the energy-momentum tensor. Thus, employing the Einstein's field equations (19) we obtain the following relations for calculation of the components of the energy-momentum tensor $T_{\mu \eta}$ :

$$
\begin{aligned}
& k T_{00}=\left(1-\lambda^{2}\right)\left(\frac{2 \lambda \lambda^{\prime}}{r}+\frac{\lambda^{2}}{r^{2}}\right), k T_{01}=k T_{10}=-\lambda\left(\frac{2 \lambda \lambda^{\prime}}{r}+\frac{\lambda^{2}}{r^{2}}\right), \\
& k T_{11}=(-1)\left(\frac{2 \lambda \lambda^{\prime}}{r}+\frac{\lambda^{2}}{r^{2}}\right), k T_{22}=\left(-r^{2}\right)\left(\lambda^{\prime 2}+\lambda \lambda^{\prime \prime}+\frac{2 \lambda \lambda^{\prime}}{r}\right), \\
& k T_{33}=\left(-r^{2} \sin ^{2} \theta\right)\left(\lambda^{\prime 2}+\lambda \lambda^{\prime \prime}+\frac{2 \lambda \lambda^{\prime}}{r}\right), \quad k=\frac{8 \pi G}{c^{4}} .
\end{aligned}
$$

For calculation of the components of the energy-momentum tensor, $T_{\mu \eta}$, by the relations (20) we should know the parameter $\lambda$ and its derivations $\lambda^{\prime}$ and $\lambda^{\prime \prime}$ for the related static field. Parameter $\lambda$ is defined by (5) as the function of the field parameters $\alpha$ and $\alpha^{\prime}$

$\lambda=\kappa\left(\alpha^{\prime}-\alpha\right) / 2=-\kappa\left(\alpha-\alpha^{\prime}\right) / 2, \quad \kappa= \pm 1$.

In order to determine the field parameters $\alpha$ and $\alpha^{\prime}$ in an electrostatic field we need to know the potential energy of the particle in that field. Thus, if a particle is an electron that is present in an electrostatic field, then the potential energy of the electron in that field, $U_{e}$, is described by the well known relation [37-39]

$$
U_{e}=q V=q A_{0} .
$$

Here $q$ is an electric charge of the electron and $V=A_{0}$ is a scalar potential of that field. For calculation of the parameter $\lambda$ in an electrostatic field we need to know the difference of the field parameters $\left(\alpha-\alpha^{\prime}\right)$, given by the general form in the first part [30] of this theory:

$$
\begin{aligned}
& \alpha_{1}-\alpha_{1}^{\prime}=\alpha_{3}-\alpha_{3}^{\prime}=2 i \sqrt{\frac{2 U}{m_{0} c^{2}}+\left(\frac{U}{m_{0} c^{2}}\right)^{2}}, \\
& \alpha_{2}-\alpha_{2}^{\prime}=\alpha_{4}-\alpha_{4}^{\prime}=-2 i \sqrt{\frac{2 U}{m_{0} c^{2}}+\left(\frac{U}{m_{0} c^{2}}\right)^{2}} .
\end{aligned}
$$

Here $m_{0}$ is a rest mass of the electron. Including the substitution $U=U_{e}$ into (23) we obtain the difference of the field parameters $\left(\alpha-\alpha^{\prime}\right)$ for an electron in an electrostatic field: 


$$
\begin{aligned}
& \alpha_{1}-\alpha_{1}^{\prime}=\alpha_{3}-\alpha_{3}^{\prime}=2 i \sqrt{\frac{2 q V}{m_{0} c^{2}}+\left(\frac{q V}{m_{0} c^{2}}\right)^{2}}, \\
& \alpha_{2}-\alpha_{2}^{\prime}=\alpha_{4}-\alpha_{4}^{\prime}=-2 i \sqrt{\frac{2 q V}{m_{0} c^{2}}+\left(\frac{q V}{m_{0} c^{2}}\right)^{2}} .
\end{aligned}
$$

Further, for the gauge field one should use the well known electrostatic ansatz [6]. Thus, including the electrostatic ansatz and applying the relations (21) and (24) we obtain the two solutions of the parameter $\lambda$ :

$$
\begin{aligned}
& A_{t}=A_{0}(r)=V(r), \quad A_{r}=A_{\theta}=A_{\phi}=0, \\
& \lambda= \pm i \kappa \sqrt{\frac{2 q V}{m_{0} c^{2}}+\left(\frac{q V}{m_{0} c^{2}}\right)^{2}}, V=A_{0}=\frac{Q}{r}, G_{e}=\frac{q}{m_{0}}, \\
& \rightarrow \lambda= \pm i \kappa \sqrt{\frac{2 G_{e} Q}{r c^{2}}+\left(\frac{G_{e} Q}{r c^{2}}\right)^{2}}, \quad \kappa= \pm 1 .
\end{aligned}
$$

Here $Q$ is a point charge of the electrostatic field, and $G_{e}$ is the Kaluza-Klein constant [7, 8].

The all items needed for calculations of the components of the energy-momentum tensor $T_{\mu \eta}$ in (20) are given by the following relations:

$$
\begin{aligned}
& \lambda^{\prime}=\mp i \kappa\left[\frac{G_{e} Q}{r^{2} c^{2}}+\left(\frac{G_{e} Q}{r^{2} c^{2}}\right)\left(\frac{G_{e} Q}{r c^{2}}\right)\right] / \sqrt{\frac{2 G_{e} Q}{r c^{2}}+\left(\frac{G_{e} Q}{r c^{2}}\right)^{2}}, \\
& \lambda \lambda^{\prime}=\left[\frac{G_{e} Q}{r^{2} c^{2}}+\left(\frac{G_{e} Q}{r^{2} c^{2}}\right)\left(\frac{G_{e} Q}{r c^{2}}\right)\right], \\
& \frac{2 \lambda \lambda^{\prime}}{r}=2\left[\frac{G_{e} Q}{r^{3} c^{2}}+\left(\frac{G_{e} Q}{r^{2} c^{2}}\right)^{2}\right], \frac{\lambda^{2}}{r^{2}}=-\left[\frac{2 G_{e} Q}{r^{3} c^{2}}+\left(\frac{G_{e} Q}{r^{2} c^{2}}\right)^{2}\right], \\
& \lambda^{\prime 2}+\lambda \lambda^{\prime \prime}=\left(\lambda \lambda^{\prime}\right)^{\prime}=-\frac{2 G_{e} Q}{r^{3} c^{2}}-3\left(\frac{G_{e} Q}{r^{2} c^{2}}\right)^{2} .
\end{aligned}
$$

Applying (26) to (18) and (20) we obtain the components of the energy-momentum tensor $T_{\mu \eta}$ and Ricci scalar $R$ in an electrostatic field:

$$
\begin{aligned}
& k T_{00}=\left(1-\lambda^{2}\right)\left(\frac{G_{e} Q}{r^{2} c^{2}}\right)^{2}=v\left(\frac{G_{e} Q}{r^{2} c^{2}}\right)^{2}, k T_{01}=k T_{10}=-\lambda\left(\frac{G_{e} Q}{r^{2} c^{2}}\right)^{2}, \\
& k=\frac{8 \pi G}{c^{4}}, k T_{11}=(-1)\left(\frac{G_{e} Q}{r^{2} c^{2}}\right)^{2}, k T_{22}=r^{2}\left(\frac{G_{e} Q}{r^{2} c^{2}}\right)^{2}, \\
& k T_{33}=\left(r^{2} \sin ^{2} \theta\right)\left(\frac{G_{e} Q}{r^{2} c^{2}}\right)^{2}, R=2\left(\lambda^{\prime 2}+\lambda \lambda^{\prime \prime}+\frac{2 \lambda \lambda^{\prime}}{r}\right)+2\left(\frac{2 \lambda \lambda^{\prime}}{r}+\frac{\lambda^{2}}{r^{2}}\right) \\
& =-2\left(\frac{G_{e} Q}{r^{2} c^{2}}\right)^{2}+2\left(\frac{G_{e} Q}{r^{2} c^{2}}\right)^{2}=0 .
\end{aligned}
$$

From the previous relation we can see that the Ricci scalar is equal to zero. Finally, included parameter $\mathrm{k}$ into the relations (27), we obtain the components of the energy-momentum tensor in an electrostatic field:

$$
\begin{aligned}
& T_{\mu \eta}=\left[T_{00}, T_{01}, T_{10}, T_{11}, T_{22}, T_{33}\right] \\
& =\left[\nu,-\lambda,-\lambda,-1, r^{2}, r^{2} \sin ^{2} \theta\right] \frac{\left(G_{e} Q\right)^{2}}{8 \pi G r^{4}}, G_{e}=\frac{q}{m_{0}} .
\end{aligned}
$$

Because the relation (28) is equal to the relation (10), the proof of the proposition 1 is finished.

(c) Remarks 1. In order to make the solution (28) consistent to the related solution in a gravitational field, we should introduce the parameter $k_{e}=8 \pi G_{e} / c^{4}$. On the other hand, for the consistence to the Maxwell field theory, this parameter should be $k_{e}=8 \pi G_{e}^{2} / c^{4}$ :

$$
\begin{array}{r}
k_{e}=\frac{8 \pi G_{e}}{c^{4}}, \rightarrow T_{\mu \eta}=\left[T_{00}, T_{01}, T_{10}, T_{11}, T_{22}, T_{33}\right] \\
=\left[\nu,-\lambda,-\lambda,-1, r^{2}, r^{2} \sin ^{2} \theta\right] \frac{\left(G_{e} Q\right)^{2}}{8 \pi G_{e} r^{4}}, \\
k_{e}=\frac{8 \pi G_{e}^{2}}{c^{4}}, \rightarrow T_{\mu \eta}=\left[T_{00}, T_{01}, T_{10}, T_{11}, T_{22}, T_{33}\right] \\
=\left[v,-\lambda,-\lambda,-1, r^{2}, r^{2} \sin ^{2} \theta\right] \frac{Q^{2}}{8 \pi r^{4}} .
\end{array}
$$

Further, the all items given by (2), (3) to (9) are also valid in a gravitational field.

(d) Proposition 2. If the gravitational static field is described by the line element (6), then the solution of the Einstein field equations gives the energy momentum tensor $T_{\mu \eta}$ of that field in the following form

$$
\begin{aligned}
T_{\mu \eta}=\left(T_{00}, T_{01}, T_{10}, T_{11}, T_{22}, T_{33}\right) \\
=\left(\nu,-\lambda,-\lambda,-1, r^{2}, r^{2} \sin ^{2} \theta\right) \frac{(G M)^{2}}{8 \pi G r^{4}} .
\end{aligned}
$$

Here $G$ and $M$ are the gravitational constant and the gravitational mass, respectively.

(e) Proof of the proposition 2. In order to prove of the proposition 2, we should start with the general relations given by (1), (2) to (21). For determination of the field parameters $\alpha$ and $\alpha^{\prime}$ in a gravitational field one need to know the potential energy of the particle in that field. Thus, if a particle with rest mass $m_{0}$ is in a gravitational field, then the potential energy of the particle in that field $U_{g}$ is described by the well known relation $[1-6]$

$$
U_{g}=m_{0} V_{g}=m_{0} A_{g 0}=-\frac{m_{0} G M}{r} \text {. }
$$

Here $V_{g}=A_{g 0}$ is a scalar potential of the gravitational static field, $G$ is the gravitational constant, $M$ is a gravitational mass, $r$ is a gravitational radius and $m_{0}$ is a rest mass of the particle that is present in a gravitational static field. For calculation of the parameter $\lambda$ in a gravitational static field we need to know the difference of the field parameters $\left(\alpha-\alpha^{\prime}\right)$, 
given in the general form by (23). Including the substitution $U$ $=U_{g}$ into (23) we obtain the difference of the field parameters $\left(\alpha-\alpha^{\prime}\right)$ for a particle in a gravitational static field:

$$
\begin{aligned}
& \alpha_{1}-\alpha_{1}^{\prime}=\alpha_{3}-\alpha_{3}^{\prime}=-2 \sqrt{\frac{2 G M}{r c^{2}}-\left(\frac{G M}{r c^{2}}\right)^{2}}, \\
& \alpha_{2}-\alpha_{2}^{\prime}=\alpha_{4}-\alpha_{4}^{\prime}=2 \sqrt{\frac{2 G M}{r c^{2}}-\left(\frac{G M}{r c^{2}}\right)^{2}} .
\end{aligned}
$$

Applying the results from (31) to the relations in (21) we obtain the two solutions of the parameter $\lambda$ in a gravitational static field

$$
\lambda=\mp \kappa \sqrt{\frac{2 G M}{r c^{2}}-\left(\frac{G M}{r c^{2}}\right)^{2}} .
$$

Including (32) to (20) we obtain the all items needed for calculations of the components of the energy-momentum tensor $T_{\mu \eta}$ in a gravitational static field

$$
\begin{aligned}
& \lambda^{\prime}=\mp \kappa\left[\frac{G M}{r^{2} c^{2}}-\left(\frac{G M}{r^{2} c^{2}}\right)\left(\frac{G M}{r c^{2}}\right)\right] / \sqrt{\frac{2 G M}{r c^{2}}-\left(\frac{G M}{r c^{2}}\right)^{2}}, \\
& \lambda \lambda^{\prime}=\left[-\frac{G M}{r^{2} c^{2}}+\left(\frac{G M}{r^{2} c^{2}}\right)\left(\frac{G M}{r c^{2}}\right)\right], \\
& \frac{2 \lambda \lambda^{\prime}}{r}=2\left[-\frac{G M}{r^{3} c^{2}}+\left(\frac{G M}{r^{2} c^{2}}\right)^{2}\right], \frac{\lambda^{2}}{r^{2}}=\left[\frac{2 G M}{r^{3} c^{2}}-\left(\frac{G M}{r^{2} c^{2}}\right)^{2}\right], \\
& \lambda^{\prime 2}+\lambda \lambda^{\prime \prime}=\left(\lambda \lambda^{\prime}\right)^{\prime}=\frac{2 G M}{r^{3} c^{2}}-3\left(\frac{G M}{r^{2} c^{2}}\right)^{2} .
\end{aligned}
$$

Now, applying the relations (33) to the equations (18) and (20) we obtain the components of the energy-momentum tensor and Ricci scalar valid for the gravitational static field:

$$
\begin{aligned}
k T_{00}= & \left(1-\lambda^{2}\right)\left(\frac{G M}{r^{2} c^{2}}\right)^{2}=v\left(\frac{G M}{r^{2} c^{2}}\right)^{2}, \\
k T_{01}= & k T_{10}=-\lambda\left(\frac{G M}{r^{2} c^{2}}\right)^{2}, k=\frac{8 \pi G}{c^{4}}, \\
k T_{11}= & (-1)\left(\frac{G M}{r^{2} c^{2}}\right)^{2}, k T_{22}=r^{2}\left(\frac{G M}{r^{2} c^{2}}\right)^{2}, \\
k T_{33}= & \left.\left(r^{2} \sin ^{2} \theta\right)\left(\frac{G M}{r^{2} c^{2}}\right)^{2}, \quad 2 \lambda \lambda^{\prime}\right)+2\left(\frac{2 \lambda \lambda^{\prime}}{r}+\frac{\lambda^{2}}{r^{2}}\right)^{2} \\
R=2 & \left(\lambda^{\prime 2}+\lambda \lambda^{\prime \prime}+\frac{2 r^{2}}{r^{2}}=0 .\right. \\
& =-2\left(\frac{G M}{r^{2} c^{2}}\right)^{2}+2\left(\frac{G M}{r^{2} c^{2}}\right)^{2}
\end{aligned}
$$

From the previous relations we can see that the Ricci scalar is equal to zero. Finally, included parameter $k$ into the relations
(34), we obtain the components of the energy-momentum tensor in the gravitational static field

$$
\begin{aligned}
T_{\mu \eta}= & {\left[T_{00}, T_{01}, T_{10}, T_{11}, T_{22}, T_{33}\right] } \\
& =\left[\nu,-\lambda,-\lambda,-1, r^{2}, r^{2} \sin ^{2} \theta\right] \frac{(G M)^{2}}{8 \pi G r^{4}} .
\end{aligned}
$$

Because the relation (35) is equal to the relation (29), the proof of the proposition 2 is finished.

(f) Remarks 2. The previous relations show that the field parameters (31) satisfy the Einstein's field equations with a cosmological constant $\Lambda=0$. In the case of a strong static gravitational field $[40-43,47]$, the quadratic term $\left(G M / r c^{2}\right)^{2}$ generates the related energy-momentum tensor $T_{\mu \eta}$ for the static field. For that case we do not need to add by hand the related energy-momentum tensor $T_{\mu \eta}$ on the right side of the Einstein's field equations. In the case of a weak static gravitational field, like in our solar system, we obtain the quadratic term $\left(G M / r c^{2}\right)^{2} \approx 0$. For that case the field parameters (31) satisfy the Einstein's field equations in a vacuum $\left(T_{\mu \eta}=0, \Lambda=0\right)$. This corresponds to the well known Schwarzschild solution of the line element.

The second interpretation could be that the quadratic term $\left(G M / r c^{2}\right)^{2}$ generates the cosmological parameter $\Lambda$ as a function of a gravitational radius [44] for $T_{\mu \eta}=0$. It has been shown in [45] that this solution of $\Lambda$ is valid for both Planck's and cosmological scales. Further, the metrics of RAF theory [32] has been applied to the derivation of the generalized relativistic Hamiltonian [36] and dynamic model of nanorobot motion in multipotential field [46].

\section{A. Proofs of the Predictions $a$ ) and $b$ ) of RAF Theory}

RAF theory predicts that: a) no a singularity at the Schwarzschild radius and b) there exists a minimal radius at $r=\left(G M / 2 c^{2}\right)$ that prevents singularity at $r=0$, i.e. the nature protects itself. In order to prove predictions a) and b) we start with the solution of the parameters $v$ and $\lambda$ in a gravitational static field given by (15) and (32) and valid for the line element (6):

$$
\begin{aligned}
& \lambda=\mp \kappa \sqrt{\frac{2 G M}{r c^{2}}\left(1-\frac{G M}{2 r c^{2}}\right)}, \kappa= \pm 1, \\
& v=1-\lambda^{2}=1-\frac{2 G M}{r c^{2}}+\left(\frac{G M}{r c^{2}}\right)^{2}=\left(1-\frac{G M}{r c^{2}}\right)^{2}, \\
& r_{\text {sch }}=\frac{2 G M}{c^{2}}, \rightarrow v_{s c h}=\frac{1}{4}, \lambda_{s c h}=\mp \kappa \sqrt{\frac{3}{4}}, \\
& r_{\text {min }}=\frac{G M}{2 c^{2}}, \rightarrow v=1, \lambda=0, r<r_{\text {min }} \rightarrow \lambda=\lambda_{\text {im }}, \\
& r \rightarrow \infty, \rightarrow v \rightarrow 1, \lambda \rightarrow 0, r=\frac{G M}{c^{2}}, \rightarrow v=0, \lambda=1 .
\end{aligned}
$$


Following the relations in (36) we can see that at the Schwarzschild radius, $r_{s c h}$, parameters $v$ and $\lambda$ are regular. This proves the prediction a) no a singularity at the Schwarzschild radius. Further, from (36), we also can see that at the minimal radius $r=r_{\text {min }}=G M / 2 c^{2}$ parameters $v$ and $\lambda$ are also regular and for $r<r_{\text {min }}$ parameter $\lambda$ becomes imaginary number $\lambda=\lambda_{\text {im }}$. This proves the prediction $b$ ) there exists a minimal radius at $r=\left(G M / 2 c^{2}\right)$ that prevents singularity at $r=0$. It seems that the existence of the minimal radius tell us that the nature protect itself from the singularity. Thus, we can say that the metrics of the line element in (6) is regular for a gravitational field in the region $r_{\text {min }} \leq r \leq \infty$. On that way the proof of the propositions a) and b) is finished.

\section{ENERGY-MOMENTUM TENSOR FOR UNIFIED FIELD}

In order to determine of the field parameters $\alpha$ and $\alpha^{\prime}$ for the unified electrical and gravitational static field we need to know the potential energy of a particle in that field. Let the source of the unified static field is an object with mass $M$, electric point charge $Q$ and radius $r$. Thus, if the particle in the unified field is an electron with rest mass $m_{0}$ and an electric charge $q$, then the potential energy of the electron in the unified field $U$ is described by the relation [1, 37-39]

$$
\begin{aligned}
U=U_{e} & +U_{g}=q V+m_{0} V_{g} \\
& =q A_{e 0}+m_{0} A_{g 0}=\frac{q Q}{r}-\frac{m_{0} G M}{r} .
\end{aligned}
$$

Here $V_{e}=A_{e 0}$ is a scalar electrical potential, $V_{g}=A_{g 0}$ is a scalar gravitational potential and $G$ is a gravitational constant. Now, following (37) we can calculate the dimensionless term $U / m_{0} c^{2}$

$$
\begin{aligned}
& \frac{U}{m_{0} c^{2}}=\frac{q Q}{m_{0} r c^{2}}-\frac{m_{0} G M}{m_{0} r c^{2}}=\frac{G_{e} Q}{r c^{2}}-\frac{G M}{r c^{2}}=\frac{M_{e g}}{r c^{2}}, \\
& G_{e}=\frac{q}{m_{0}}, M_{e g}=G_{e} Q-G M .
\end{aligned}
$$

Here parameter $G_{e}=q / m_{0}$ is a constant well known in Kaluza-Klein theory [7-14]. The four solutions of the field parameters $\alpha$ and $\alpha^{\prime}$ for the electron in the unified electrical and gravitational static field can be obtained by the substitution of the dimensionless term (38) into the general solution of the field parameters $\alpha$ and $\alpha^{\prime}$, given in the first part [30] of this theory:

$$
\begin{gathered}
f(U)=2 U / m_{0} c^{2}+\left(U / m_{0} c^{2}\right)^{2} \\
=2 M_{e g} / r c^{2}+\left(M_{e g} / r c^{2}\right)^{2}, \rightarrow \\
\alpha_{1,2}=1 \pm i \sqrt{f(U)}, \alpha_{1,2}^{\prime}=1 \mp i \sqrt{f(U)}, \\
\alpha_{3,4}=-1 \pm i \sqrt{f(U)}, \alpha_{3,4}^{\prime}=-1 \mp i \sqrt{f(U)} .
\end{gathered}
$$

It is easy to prove that the all $\alpha \alpha^{\prime}$ pairs from (39) satisfy the following invariant relations:

$$
\begin{gathered}
\alpha_{i} \alpha_{i}^{\prime}=\left(1+\frac{U}{m_{0} c^{2}}\right)^{2}=\left(1+\frac{q Q}{m_{0} r c^{2}}-\frac{m_{0} G M}{m_{0} r c^{2}}\right)^{2} \\
=\left(1+\frac{M_{e g}}{r c^{2}}\right)^{2}=\alpha \alpha^{\prime}, i=1,2,3,4, \\
E_{c}=m_{0} c^{2} \sqrt{\alpha \alpha^{\prime}}=m_{0} c^{2}\left(1+\frac{q Q}{m_{0} r c^{2}}-\frac{m_{0} G M}{m_{0} r c^{2}}\right) \\
=m_{0} c^{2}+q V_{e}+m_{0} V_{g} .
\end{gathered}
$$

Here $E_{c}$ is the covariant energy of an electron standing $(v=0)$ in the unified electrical and gravitational static field. For calculation some of the quantities in that field we often need to know the difference of the field parameters $\left(\alpha-\alpha^{\prime}\right)$ for an electron in the unified electrical and gravitational static field:

$$
\begin{aligned}
& \alpha_{1}-\alpha_{1}^{\prime}=\alpha_{3}-\alpha_{3}^{\prime}=2 i \sqrt{\frac{2 M_{e g}}{m_{0} c^{2}}+\left(\frac{M_{e g}}{m_{0} c^{2}}\right)^{2}}, \\
& \alpha_{2}-\alpha_{2}^{\prime}=\alpha_{4}-\alpha_{4}^{\prime}=-2 i \sqrt{\frac{2 M_{e g}}{m_{0} c^{2}}+\left(\frac{M_{e g}}{m_{0} c^{2}}\right)^{2}} .
\end{aligned}
$$

The all items given by (2), (3) to (9) are also valid for the unified electrical and gravitational static field.

(g) Proposition 3. Let the source of the unified electrical and gravitational static field is an object with mass $M$, electric point charge $Q$ and radius $r$. Further, let a particle is an electron with rest mass $m_{0}$ and an electric charge $q$ that is present in this unified electrical end gravitational static field. If the unified field is described by the line element (6), then the solution of the Einstein field equations gives the energy momentum tensor, $T_{\mu \eta}$, valid for that field

$$
\begin{aligned}
T_{\mu \eta}= & \left(T_{00}, T_{01}, T_{10}, T_{11}, T_{22}, T_{33}\right) \\
& =\left(v,-\lambda,-\lambda,-1, r^{2}, r^{2} \sin ^{2} \theta\right) \frac{M_{e g}^{2}}{8 \pi G r^{4}}, \\
M_{e g}= & G_{e} Q-G M, \quad G_{e}=\frac{q}{m_{0}} .
\end{aligned}
$$

Parameter $G_{e}$ is a constant that remands us to the constant of motion in the geodesic equation of the Kaluza-Klein theory [7-14] and $G$ is the gravitational constant.

(h) Proof of the proposition 3. In order to prove of the proposition 3 , we should start with the general relations given by (1), (2) to (21). Thus, applying (41) to (21) we obtain two solutions of the parameter $\lambda$, valid in the unified static field

$$
\lambda= \pm i \kappa \sqrt{\frac{2 M_{e g}}{r c^{2}}+\left(\frac{M_{e g}}{r c^{2}}\right)^{2}} .
$$

The all items needed for calculations of the components of the energy-momentum tensor $T_{\mu \eta}$ in (20) are given by the following relations: 


$$
\begin{aligned}
& \lambda^{\prime}=\mp i \kappa\left[\frac{M_{e g}}{r^{2} c^{2}}+\left(\frac{M_{e g}}{r^{2} c^{2}}\right)\left(\frac{M_{e g}}{r c^{2}}\right)\right] / \sqrt{\frac{2 M_{e g}}{r c^{2}}+\left(\frac{M_{e g}}{r c^{2}}\right)^{2}}, \\
& \lambda \lambda^{\prime}=\left[\frac{M_{e g}}{r^{2} c^{2}}+\left(\frac{M_{e g}}{r^{2} c^{2}}\right)\left(\frac{M_{e g}}{r c^{2}}\right)\right], \\
& \frac{2 \lambda \lambda^{\prime}}{r}=2\left[\frac{M_{e g}}{r^{3} c^{2}}+\left(\frac{M_{e g}}{r^{2} c^{2}}\right)^{2}\right], \frac{\lambda^{2}}{r^{2}}=-\left[\frac{2 M_{e g}}{r^{3} c^{2}}+\left(\frac{M_{e g}}{r^{2} c^{2}}\right)^{2}\right], \\
& \lambda^{\prime 2}+\lambda \lambda^{\prime \prime}=\left(\lambda \lambda^{\prime}\right)^{\prime}=-\frac{2 M_{e g}}{r^{3} c^{2}}-3\left(\frac{M_{e g}}{r^{2} c^{2}}\right)^{2} .
\end{aligned}
$$

Now, applying (43) to (18) and (20), we obtain the components of the energy-momentum tensor $k T_{\mu \eta}$ and Ricci scalar $R$ of the unified static field:

$$
\begin{aligned}
& k T_{00}=\left(1-\lambda^{2}\right)\left(\frac{M_{e g}}{r^{2} c^{2}}\right)^{2}=v\left(\frac{M_{e g}}{r^{2} c^{2}}\right)^{2}, k=\frac{8 \pi G}{c^{4}}, \\
& k T_{01}=k T_{10}=-\lambda\left(\frac{M_{e g}}{r^{2} c^{2}}\right)^{2}, k T_{11}=(-1)\left(\frac{M_{e g}}{r^{2} c^{2}}\right)^{2}, \\
& k T_{22}= r^{2}\left(\frac{M_{e g}}{r^{2} c^{2}}\right)^{2}, k T_{33}=\left(r^{2} \sin ^{2} \theta\right)\left(\frac{M_{e g}}{r^{2} c^{2}}\right)^{2}, \\
& R=2\left(\lambda^{\prime 2}+\lambda \lambda^{\prime \prime}+\frac{2 \lambda \lambda^{\prime}}{r}\right)+2\left(\frac{2 \lambda \lambda^{\prime}}{r}+\frac{\lambda^{2}}{r^{2}}\right)^{2} \\
&=-2\left(\frac{M_{e g}}{r^{2} c^{2}}\right)^{2}+2\left(\frac{M_{e g}}{r^{2} c^{2}}\right)^{2}=0 .
\end{aligned}
$$

From the previous relations we can see that the Ricci scalar is equal to zero. Finally, included parameter $\mathrm{k}$ into the relations (45), we obtain the components of the energy-momentum tensor $T_{\mu \eta}$ in the unified electrical and gravitational static field

$$
\begin{aligned}
T_{\mu \eta}=\left[T_{00}, T_{01}, T_{10}, T_{11}, T_{22}, T_{33}\right] \\
=\left[\nu,-\lambda,-\lambda,-1, r^{2}, r^{2} \sin ^{2} \theta\right] \frac{M_{e g}^{2}}{8 \pi G r^{4}} .
\end{aligned}
$$

Because (46) is equal to (42), we conclude that the proof of the proposition 3 is finished.

(i) Remarks 3. The energy momentum tensor (46) is general in the following sense: a) putting $M_{e g}=G_{e} Q$ one obtains the solution in an electrostatic field, b) putting $M_{e g}=$ $G M$ one obtains the solution in a gravitational static field. Using the dimensional analysis, $\operatorname{dim}\left(\left(G_{e}=q / m_{0}\right)^{2}\right)=$ $\operatorname{dim}\left((\sqrt{ } G)^{2}\right)=\operatorname{dim}(G)$, the equation (28) can be transformed into the new form:

$$
\begin{aligned}
T_{\mu \eta} & =\left[T_{00}, T_{01}, T_{10}, T_{11}, T_{22}, T_{33}\right] \\
& =\left[\nu,-\lambda,-\lambda,-1, r^{2}, r^{2} \sin ^{2} \theta\right] K \frac{(Q)^{2}}{8 \pi r^{4}}, \\
G_{e} & =\frac{q}{m_{0}}, \quad K=\frac{G_{e}^{2}}{G}, \quad \operatorname{dim}(K)=1 .
\end{aligned}
$$

Sometimes (see [6]), the components of the energy-momentum tensor $T_{\mu \eta}$ in an electrostatic field have been described by the relations (47).

\section{CONCLUSION}

In this paper we proved that the field parameters $\alpha$ and $\alpha^{\prime}$ of the electrical, gravitational and unified fields satisfy the Einstein's field equations and automatically generate the related energy-momentum tensor in the standard four dimensions (4D). This means that for electrical, gravitational and unified fields we do not need to add by hand the energy-momentum tensor to the right side of the Einstein's field equations. In a strong static gravitational field the quadratic term $\left(G M / r c^{2}\right)^{2}$ generates the energy - momentum tensor on the right side of the Einstein's field equations. In the case of a weak static gravitational field, like in our solar system, we obtain the quadratic term $\left(G M / r c^{2}\right)^{2}$ close to zero. For that case the field parameters satisfy the Einstein's field equations in a vacuum $\left(T_{\mu \eta}=0, \Lambda=0\right)$. This corresponds to the well known Schwarzschild solution of the line element. Further, we also proved two predictions of RAF theory: a) no a singularity at the Schwarzschild radius, b) there exists a minimal radius at $r=\left(G M / 2 c^{2}\right)$ that prevents singularity at $r$ $=0$, i.e. the nature protects itself. The predictions c) and d) are considered in the third part of the theory. If RAF theory is correct, then it could be applied to the both weak and strong fields at the Universe and Planck's scales giving the new light to the regions like black holes, quantum theory, high energy physics, Big Bang theory and cosmology.

\section{ACKNOWLEDGMENTS}

The author wishes to thank to the anonymous reviewers for a variety of helpful comments and suggestions. This work is supported by grants (120-1201842-3048) from the National Scientific Foundation of Republic of Croatia.

\section{REFERENCES}

[1] A. Einstein, Ann. Phys. 49, 769-822 (1916).

[2] A. Einstein, The Meaning of Relativity (Princeton Univ. Press, Princeton, 1955).

[3] C. Sean, Spacetime and Geometry: An introduction to General Relativiry (Amazon.com Books.htm, Hardcover, 2003).

[4] S. Weinberg, Gravitation and Cosmology: Principles and Application of the General Theory of Relativity (Gebundene Ausgabe, RelEspWeinberg.pdf., 1972).

[5] S. W. Hawking, G. F. R. Ellis, The Large Scale Structure of Space-Time (Univ. Press, Cambridge, 1973).

[6] M. Blau, Lecture Notes on General Relativity (A. Einstein Center for Fundamental Physics, Univ. Bern, Bern, 2012, 2014).

[7] T. Kaluza, Zum Unitätsproblem in der Physik (Sitzungsber. Preuss. Akad. Wiss., Berlin, 1921).

[8] O. Klein, Z. Phys. A 37, 895-906 (1926).

[9] E. Witten, Nucl. Phys. B 186, 412-428 (1981).

[10] T. Appelquist, A. Chodos, and P. G. O. Freund, Modern Kaluza-Klein Theories (Addison-Wesley, Menlo Park, Cal., 1987). 
[11] M. J. Duff, Kaluza-Klein Theory in Perspective (Proc. of the Symposium: The Oskar Klein Centenary, World Scientific, Singapore, 22-35, 1994).

[12] J. M. Overduin, and P. S. Wesson, Phys. Rep. 283, 303-378 (1997).

[13] P. S. Wesson, Space-Time-Matter, Modern Kaluza - Klein Theory (World Scientific, Singapore, 1999).

[14] P. S.Wesson, Five-Dimensional Physics: Classical and Quantum Consequences of Kaluza-Klein Cosmology (World Scientific, Singapore, 2006).

[15] D. Z. Freedman, and A. Van Proeyen, Supergravity (Cambridge Univ. Press, Cambridge, 2012)

[16] J. Wess, B. and A. Zumino, Phys. Lett. B 49, 52 (1974)

[17] M. K. Gaillard, and B. Zumino, Nucl. Phys. B 193, 221 (1981).

[18] M. B. Green, J. H. Schwarz, and E. Witten, Superstring Theory (Cambridge Univ. Press, Cambridge, 1987).

[19] J. Polchinski, String Theory (Cambridge Univ. Press, Cambridge, 1998).

[20] R. Brandenberger, and C. Vafa, Nucl. Phys. B 316, 391-410 (1989).

[21] N. Arkani-Hamed, A. G. Cohen, and H. Georgi, Phys. Rev. Lett. 86, 4757 (2001)

[22] C. T. Hill, S. Pokorski, and J. Wang, Phys. Rev. D 64, 105005 (2001).

[23] C. Cshaki, G. D. Kribs, and J. Terning, Phys. Rev. D 65, 015004 (2002)

[24] E. C. Poggio, H. R. Quinn, and S. Weinberg, Phys. Rev. D 13, 1958 (1976).

[25] T. R. Taylor, and G. Veneziano, Phys. Lett. B 212, 147 (1988).

[26] H. C. Cheng, B. A. Dobrescu, and C. T. Hill, Nucl. Phys. B 573, 597 (2000).

[27] C. Cshaki, J. Erlich, C. Grojean, and G. D. Kribs, Phys. Rev. D 65, 015003 (2002)

[28] N. Arkani-Hamed, and M. Schmaltz, Phys. Rev. D 61, 033005 (2000).

[29] M. Gogberashvili, Europhys. Lett. 49, 396 (2000).

[30] B. M. Novakovic, Relativistic alpha field theory - Part I. To be published in IJNTR (2015).

[31] B. M. Novakovic, Relativistic alpha field theory-Part III. To be published in IJNTR (2015).

[32] B. M. Novakovic, Int. J. of Comput. Anticip. Syst., IJCAS 27, p. 93 (2014).

[33] S. Gallot, D. Hullin, and D. J. Lafontane, Riemannian Geometry ( Springer-Verlag, Berlin, New York, ed. 3, 2004).

[34] C. T. J. Dodson, and T. Poston, Tensor Geometry. Graduate Texts in Mathematics (Springer-Verlag, Berlin, New York, ed. 2, 1991), p. 130

[35] M. T. Vaughin, Introduction to Mathematical Physics (Wiley-VCH Verlag GmbH \& Co., Weinheim, 2007)

[36] B. M. Novakovic, in Proceedings of the Ninth Int. Conf. on Comp. Anticip. Syst., Liege, 2009, edited by D. Dubois (University of Liege, Liege 2009), AIP-CP 1303, p. 141 (2010). DOI: 10.1063/1.3527149.

[37] P. A. M. Dirac, Directions in Physics (Wiley, New York, 1978).

[38] I. Supek, Theoretical Physics and Structure of Matter, Part I (Skolska knjiga, Zagreb, 1992)

[39] I. Supek, Theoretical Physics and Structure of Matter, Part II (Skolska knjiga, Zagreb, 1990).

[40] D. H. Perkins, Introduction to High Energy Physics (Cambridge Univ. Press, Cambridge, 2000).

[41] D. Sherman, et al., Nat. Phys. 11, 1-5 (2015).

[42] J. Steinhauer, Nat. Phys. 10, 864-869 (2014)

[43] M. Meckel, et al. Nat. Phys. 10, 594 - 600 (2014)

[44] B. M. Novakovic, D. Novakovic, and A. Novakovic. in Proceedings of the Sixth Int. Conf. on Comp. Anticip. Syst., Liege, 2003, edited by D. Dubois (University of Liege, Liege 2003), AIP-CP 718, p.133 (2004). DOI: $10.1063 / 1.1787318$

[45] B. M. Novakovic, D. Novakovic, and A. Novakovic, in Proceedings of the Seventh Int. Conf. on Comp. Anticip. Syst., Liege, 2005, edited by D. Dubois (University of Liege, Liege 2005), AIP-CP 839, p. 144, (2006). DOI: 10.1063/1.2216624.

[46] B. M. Novakovic, Strojarstvo 53 (2), 103-111 (2011).

[47] R. Ding, et al. Phys. Rev. D 92, (015008) (2015).

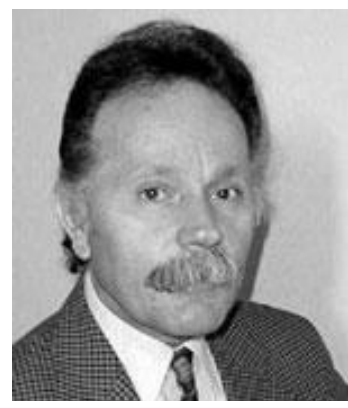

Branko Novakovic is a Professor emeritus at FSB - University of Zagreb, Croatia. Prof. Novakovic received his $\mathrm{PhD}$ from the University of Zagreb in 1978. His research of interest includes physics, control systems, robotics, neural networks, and fuzzy control. He is author of two books, Control Systems (1985) and Control Methods in Robotics, Flexible Manufacturing Systems and Processes (1990), and co-author of a book Artificial Neural Networks (1998). He has published over 220 research papers in his research of interest. 